\title{
INFINITELY DIVISIBLE METRICS AND CURVATURE INEQUALITIES FOR OPERATORS IN THE COWEN-DOUGLAS CLASS
}

\author{
SHIBANANDA BISWAS, DINESH KUMAR KESHARI, AND GADADHAR MISRA
}

\begin{abstract}
The curvature $\mathcal{K}_{T}(w)$ of a contraction $T$ in the Cowen-Douglas class $B_{1}(\mathbb{D})$ is bounded above by the curvature $\mathcal{K}_{S^{*}}(w)$ of the backward shift operator. However, in general, an operator satisfying the curvature inequality need not be contractive. In this note, we characterize a slightly smaller class of contractions using a stronger form of the curvature inequality. Along the way, we find conditions on the metric of the holomorphic Hermitian vector bundle $E_{T}$ corresponding to the operator $T$ in the Cowen-Douglas class $B_{1}(\mathbb{D})$ which ensures negative definiteness of the curvature function. We obtain a generalization for commuting tuples of operators in the class $B_{1}(\Omega)$, for a bounded domain $\Omega$ in $\mathbb{C}^{m}$.
\end{abstract}

\section{INTRODUCTION}

Let $\mathcal{H}$ be a complex separable Hilbert space and $\mathcal{L}(\mathcal{H})$ denote the collection of bounded linear operators on $\mathcal{H}$. The following important class of operators was introduced in [3].

Definition 1.1. For a connected open subset $\Omega$ of $\mathbb{C}$ and a positive integer $n$, let

$$
\begin{aligned}
& B_{n}(\Omega)=\{\quad T \in \mathcal{L}(\mathcal{H}) \mid \Omega \subset \sigma(T), \\
& \operatorname{ran}(T-w)=\mathcal{H} \text { for } w \in \Omega \text {, } \\
& \bigvee_{w \in \Omega} \operatorname{ker}(T-w)=\mathcal{H} \text {, } \\
& \operatorname{dim} \operatorname{ker}(T-w)=n \text { for } w \in \Omega\},
\end{aligned}
$$

where $\sigma(T)$ denotes the spectrum of the operator $T$.

We recall (cf. [3]) that an operator $T$ in the class $B_{n}(\Omega)$ defines a holomorphic Hermitian vector bundle $E_{T}$ in a natural manner. It is the sub-bundle of the trivial bundle $\Omega \times \mathcal{H}$ defined by

$$
E_{T}=\{(w, x) \in \Omega \times \mathcal{H}: x \in \operatorname{ker}(T-w)\}
$$

with the natural projection map $\pi: E_{T} \rightarrow \Omega, \pi(w, x)=w$. It is shown in [3, Proposition 1.12] that the mapping $w \longrightarrow \operatorname{ker}(T-w)$ defines a rank $n$ holomorphic Hermitian vector bundle $E_{T}$ over $\Omega$ for $T \in B_{n}(\Omega)$. In [3] it was also shown that the equivalence class of the holomorphic Hermitian vector bundle $E_{T}$ and the unitary equivalence class of the operator $T$ determine each other.

Theorem 1.2. The operators $T$ and $\widetilde{T}$ in $B_{n}(\Omega)$ are unitarily equivalent if and only if the corresponding holomorphic Hermitian vector bundles $E_{T}$ and $E_{\widetilde{T}}$ are equivalent.

2000 Mathematics Subject Classification. 47B32, 47B35.

Key words and phrases. Cowen-Douglas class, curvature inequality, holomorphic Hermitian vector bundle, kernel function, infinite divisibility.

The work of S. Biswas was supported by a postdoctoral fellowship funded by the Skirball Foundation via the Center for Advanced Studies in Mathematics at Ben-Gurion University of the Negev. The work of D.K. Keshari was supported in the form of a CSIR Research Fellowship at the Indian Institute of Science. The work of G. Misra was supported in part by UGC - SAP and by a grant from the Department of Science and Technology. 
In general, it is not easy to decide if two holomorphic Hermitian vector bundles are equivalent except when the rank of the bundle is 1 . In this case, the curvature

$$
\mathcal{K}(w)=-\frac{\partial^{2} \log \|\gamma(w)\|^{2}}{\partial w \partial \bar{w}},
$$

of the line bundle $E$, defined with respect to a non-zero holomorphic section $\gamma$ of $E$, is a complete invariant. The definition of the curvature is independent of the choice of the section $\gamma$ : If $\gamma_{0}$ is another holomorphic section of $E$, then $\gamma_{0}=\phi \gamma$ for some non-zero holomorphic function $\phi$ defined on an open subset $\Omega_{0}$ of $\Omega$, consequently the harmonicity of log $|\phi|$, completes the verification.

Let $T \in B_{1}(\Omega)$. Fix $w \in \Omega$ and let $\gamma$ be a holomorphic section of the line bundle $E_{T}$. From [3, Lemma 1.22], it follows that the vectors $\gamma(w)$ and $\partial \gamma(w)$ from a basis of $\operatorname{ker}(T-w)^{2}$. Let $N_{T}(w)=\left.T\right|_{\operatorname{ker}(T-w)^{2}}$ and $\left\{\gamma_{1}(w), \gamma_{2}(w)\right\}$ be the basis obtained by applying Gram-Schmidt orthonormalization to the vectors $\gamma(w)$ and $\partial \gamma(w)$. The linear transformation $N_{T}(w)$ has the matrix representation

$$
N_{T}(w)=\left(\begin{array}{cc}
w & h_{T}(w) \\
0 & w
\end{array}\right)
$$

where $h_{T}(w)=\left(-\mathcal{K}_{T}(w)\right)^{-\frac{1}{2}}$, with respect to the orthonormal basis $\left\{\gamma_{1}(w), \gamma_{2}(w)\right\}$.

The curvature $\mathcal{K}_{T}(w)$ of an operator $T$ in $B_{1}(\Omega)$ is negative. To see this, recall that the curvature may also be expressed (cf.[3, page - 195]) in the form

$$
\mathcal{K}_{T}(w)=-\frac{\|\gamma(w)\|^{2}\left\|\gamma^{\prime}(w)\right\|^{2}-\left|\left\langle\gamma^{\prime}(w), \gamma(w)\right\rangle\right|^{2}}{\|\gamma(w)\|^{4}} .
$$

Applying Cauchy - Schwarz inequality, we see that the numerator is positive.

Let $\left\{e_{0}, e_{1}\right\}$ be an orthonormal set of vectors. Suppose $N$ is a nilpotent linear transformation defined by the rule

$$
e_{1} \rightarrow a e_{0}, e_{0} \rightarrow 0, a \in \mathbb{C} .
$$

Then $|a|$ determines the unitary equivalence class of $N$.

The localization $N_{T}(w)-w I_{2}=\left(\begin{array}{cc}0 & h_{T}(w) \\ 0 & 0\end{array}\right)$ of the operator $T$ in $B_{1}(\Omega)$ is nilpotent. Now, $h_{T}(w)>0$ since we have shown that the curvature $\mathcal{K}_{T}(w)$ is negative. Hence the curvature $\mathcal{K}_{T}(w)$ is an invariant for the operator $T$. The non-trivial converse of this statement follows from Theorem 1.2. Thus the operators $T$ and $\widetilde{T}$ in $B_{1}(\Omega)$ are unitarily equivalent if and only if $N_{T}(w)$ is unitarily equivalent to $N_{\widetilde{T}}(w)$ for $w$ in $\Omega$.

Note that if $T \in B_{1}(\mathbb{D})$ is a contraction, that is, $\|T\| \leq 1$, then $N_{T}(w)$ is a contraction for each $w \in \mathbb{D}$. Observe that $\left(\begin{array}{ll}a & c \\ 0 & b\end{array}\right)$ is a contraction if and only if $|a| \leq 1$ and $|c|^{2} \leq\left(1-|a|^{2}\right)\left(1-|b|^{2}\right)$. Thus $\left\|N_{T}(w)\right\| \leq 1$ if and only if $\mathcal{K}_{T}(w) \leq-\frac{1}{\left(1-|w|^{2}\right)^{2}}, w \in \mathbb{D}$. The adjoint $S^{*}$ of the unilateral shift operator $S$ is in $B_{1}(\mathbb{D})$. It is easy to see that $\gamma_{S^{*}}(w)=\left(1, w, \ldots, w^{n}, \ldots\right) \in \ell_{+}^{2}, w \in \mathbb{D}$, is a holomorphic section for the corresponding holomorphic Hermitian line bundle $E_{S^{*}}$. The norm $\left\|\gamma_{S^{*}}(w)\right\|^{2}$ of the section $\gamma_{S^{*}}$ is $\left(1-|w|^{2}\right)^{-1}$ and hence the curvature $\mathcal{K}_{S^{*}}(w)$ of the operator $S^{*}$ is given by the formula $-\frac{1}{\left(1-|w|^{2}\right)^{2}}, w \in \mathbb{D}$. We have therefore proved:

Proposition 1.3. If $T$ is a contractive operator in $B_{1}(\mathbb{D})$, then the curvature of $T$ is bounded above by the curvature of the backward shift operator $S^{*}$.

We think of the operator $S^{*}$ as an extremal operator within the class of contractions in $B_{1}(\mathbb{D})$. This is a special case of the curvature inequality proved in [8]. The curvature inequality is equivalent to contractivity of the operators $N_{T}(w), w \in \Omega$, while the contractivity of the operator $T$ is global in nature. So, it is natural to expect that the validity of the inequality $\mathcal{K}_{T}(w) \leq-\frac{1}{\left(1-|w|^{2}\right)^{2}}, w \in \mathbb{D}$, need not force $T$ to be a contraction. Indeed, J. Agler had 
communicated the existence of an operator $T,\|T\|>1$, in $B_{1}(\mathbb{D})$ with $\mathcal{K}_{T}(w) \leq \mathcal{K}_{S^{*}}(w)$ (cf. [ $[$, Note added in proof]) to G. Misra. Unfortunately, there is a printing error in [8, Note added in proof], which should be corrected by reversing the inequality sign. No explicit example has been written down of this phenomenon. We provide such an example here.

The main point of this note is to investigate additional conditions on the curvature, apart from the inequality we have discussed above, which will ensure contractivity. We give an alternative proof the curvature inequality. A stronger inequality becomes apparent from this proof. It is this stronger inequality which, as we will show below, admits a converse.

An operator $T$ in the class $B_{1}(\Omega)$, as is well-known (cf. [3, pp. 194 ]), is unitarily equivalent to the adjoint $M^{*}$ of the multiplication operator $M$ by the co-ordinate function on some Hilbert space $\mathcal{H}_{K}$ of holomorphic functions on $\Omega^{*}:=\{z \in \mathbb{C}: \bar{z} \in \Omega\}$ possessing a reproducing kernel $K$.

The kernel $K$ is a complex valued function defined on $\Omega^{*} \times \Omega^{*}$ which is holomorphic in the first variable and anti-holomorphic in the second. In consequence, the map $\bar{w} \rightarrow K(\cdot, w), w \in \Omega^{*}$, is holomorphic on $\Omega$. We have $K(z, w)=\overline{K(w, z)}$ making it Hermitian. It is positive definite in the sense that the $n \times n$ matrix

$$
\left(\left(K\left(w_{i}, w_{j}\right)\right)\right)_{i, j=1}^{n}
$$

is positive definite for every subset $\left\{w_{1}, \ldots, w_{n}\right\}$ of $\Omega^{*}, n \in \mathbb{N}$. Finally, the kernel $K$ reproduces the value of functions in $\mathcal{H}_{K}$, that is, for any fixed $w \in \Omega^{*}$, the holomorphic function $K(\cdot, w)$ belongs to $\mathcal{H}_{K}$ and

$$
f(w)=\langle f, K(\cdot, w)\rangle, f \in \mathcal{H}_{K}, w \in \Omega^{*} .
$$

The correspondence between the operator $T$ in $B_{1}(\Omega)$ and the operator $M^{*}$ on the Hilbert space $\mathcal{H}_{K}$ is easy to describe (cf. [3, pp. 194 ]). Let $\gamma$ be a non-zero holomorphic section (for bounded domain in $\mathbb{C}$, by Grauert's Theorem, a global section exists) for the operator $T$ acting on the Hilbert space $\mathcal{H}$. Consider the map $\Gamma: \mathcal{H} \rightarrow \mathcal{O}\left(\Omega^{*}\right)$, where $\mathcal{O}\left(\Omega^{*}\right)$ is the space of holomorphic functions on $\Omega^{*}$, defined by $\Gamma(x)(z)=\langle x, \gamma(\bar{z})\rangle, z \in \Omega^{*}$. Transplant the inner product from $\mathcal{H}$ on the range of $\Gamma$. The map $\Gamma$ is now unitary from $\mathcal{H}$ onto the completion of $\operatorname{ran} \Gamma$. Define $K$ to be the function $K(z, w)=\Gamma(\gamma(\bar{w}))(z)=\langle\gamma(\bar{w}), \gamma(\bar{z})\rangle, z, w \in \Omega^{*}$. Set $K_{w}(\cdot):=K(\cdot, w)$. Thus $K_{w}$ is the function $\Gamma(\gamma(\bar{w}))$. It is then easily verified that $K$ has the reproducing property, that is,

$$
\begin{aligned}
\langle\Gamma(x)(z), K(z, w)\rangle_{\operatorname{ran} \Gamma} & =\left\langle(\langle x, \gamma(\bar{z})\rangle),(\langle\gamma(\bar{w}), \gamma(\bar{z}))\rangle_{\operatorname{ran} \Gamma}\right. \\
& =\langle\Gamma x, \Gamma(\gamma(\bar{w}))\rangle_{\operatorname{ran} \Gamma}=\langle x, \gamma(\bar{w})\rangle_{\mathcal{H}} \\
& =\Gamma(x)(w), x \in \mathcal{H}, w \in \Omega^{*}
\end{aligned}
$$

It follows that $\left\|K_{w}(\cdot)\right\|^{2}=K(w, w), w \in \Omega^{*}$. Also, $K_{w}(\cdot)$ is an eigenvector for the operator $\Gamma T \Gamma^{*}$ with eigenvalue $\bar{w}$ in $\Omega$ :

$$
\begin{aligned}
\Gamma T \Gamma^{*}\left(K_{w}(\cdot)\right) & =\Gamma T \Gamma^{*}(\Gamma(\gamma(\bar{w}))) \\
& =\Gamma T \gamma(\bar{w}) \\
& =\Gamma \bar{w} \gamma(\bar{w}) \\
& =\bar{w} K_{w}(\cdot), w \in \Omega^{*} .
\end{aligned}
$$

Since the linear span of the vectors $\left\{K_{w}: w \in \Omega^{*}\right\}$ is dense in $\mathcal{H}_{K}$, it follows that $\Gamma T \Gamma^{*}$ is the adjoint $M^{*}$ of the multiplication operator $M$ on $\mathcal{H}_{K}$. We therefore assume, without loss of generality, that an operator $T$ in $B_{1}(\Omega)$ has been realized as the adjoint $M^{*}$ of the multiplication operator $M$ on some Hilbert space $\mathcal{H}_{K}$ of holomorphic functions on $\Omega^{*}$ possessing a reproducing kernel $K$. 
Remark 1.4. The contractivity of the adjoint $M^{*}$ of the multiplication operator $M$ on some reproducing kernel Hilbert space $\mathcal{H}_{K}$ is equivalent to the requirement that $K^{\ddagger}(z, w):=(1-$ $z \bar{w}) K(z, w)$ is positive definite on $\mathbb{D}$ (cf. [1, Corollary 2.37] and [7, Lemma 1]). Suppose that the operator $M^{*}$ is in $B_{1}(\mathbb{D})$. Here is a second proof of the curvature inequality:

We have

$$
\frac{\partial^{2}}{\partial w \partial \bar{w}} \log K(w, w)=\frac{\partial^{2}}{\partial w \partial \bar{w}} \log \frac{1}{\left(1-|w|^{2}\right)}+\frac{\partial^{2}}{\partial w \partial \bar{w}} \log K^{\ddagger}(w, w), w \in \mathbb{D},
$$

which we rewrite as

$$
\mathcal{K}_{M^{*}}(w)=\mathcal{K}_{S^{*}}(w)-\frac{\partial^{2}}{\partial w \partial \bar{w}} \log K^{\ddagger}(w, w), w \in \mathbb{D} .
$$

Recalling that $\frac{\partial^{2}}{\partial w \partial \bar{w}} \log K^{\ddagger}(w, w)$ must be positive (see (1.1)) as long as $K^{\ddagger}$ is positive definite, we conclude that

$$
\mathcal{K}_{M^{*}}(w) \leq \mathcal{K}_{S^{*}}(w), w \in \mathbb{D} .
$$

The fibre at $\bar{w}$ of the holomorphic bundle $E_{M^{*}}$ for $M^{*}$ in $B_{1}(\Omega)$ is the one-dimensional kernel at $\bar{w}$ of the operator $M^{*}$ spanned by $K_{w}(\cdot), w \in \Omega^{*}$. In general, there is no obvious way to define an inner product between the two vectors $K_{w}(\cdot)$ and $\left(\frac{\partial}{\partial \bar{w}} K_{w}\right)(\cdot)$. However since these vectors belong to the same Hilbert space (cf. [5, Lemma 4.3]), in our special case, there is a natural inner product defined between them. This ensures, via the Cauchy-Schwarz inequality, the negativity of the curvature $\mathcal{K}_{T}$. The reproducing kernel function $K$ of the Hilbert space $\mathcal{H}_{K}$ encodes the mutual inner products of the vectors $\left\{K_{w}(\cdot): w \in \Omega^{*}\right\}$. The Cauchy-Schwarz inequality, in turn, is just the positivity of the Gramian of the two vectors $K_{w}(\cdot)$ and $\left(\frac{\partial}{\partial \bar{w}} K_{w}\right)(\cdot)$, $w \in \Omega^{*}$. The positive definiteness of $K$ is a much stronger positivity requirement involving all the derivatives of the holomorphic section $K_{w}(\cdot)$ defined on $\Omega^{*}$. We exploit this to show that the curvature function $\left(\frac{\partial^{2}}{\partial z \partial \bar{w}} \log K\right)(z, w)$ is actually negative definite not just negative, whenever $K^{t}$ is assumed to be positive definite for all $t>0$.

We now construct an example of an operator which is not contractive but its curvature is dominated by the curvature of the backward shift. Expanding the function $K(z, w)=\frac{8+8 z \bar{w}-z^{2} \bar{w}^{2}}{1-z \bar{w}}$ in $z \bar{w}$, we see that it has the form $8+16 z \bar{w}+15 \frac{z^{2} \bar{w}^{2}}{1-z \bar{w}}$. Therefore, it defines a positive definite kernel on the unit disk $\mathbb{D}$. The monomials $\frac{z^{n}}{\left\|z^{n}\right\|}$ with $\|1\|^{2}=\frac{1}{8},\|z\|^{2}=\frac{1}{16}$ and $\left\|z^{n}\right\|^{2}=\frac{1}{15}$ for $n \geq 2$ forms an orthonormal basis in the corresponding Hilbert space $\mathcal{H}_{K}$. The multiplication operator $M$ maps $\frac{z^{n}}{\left\|z^{n}\right\|}$ to $\frac{\left\|z^{n+1}\right\|}{\left\|z^{n}\right\|} \frac{z^{n+1}}{\left\|z^{n+1}\right\|}$. Hence it corresponds to a weighted shift operator $W$ with the weight sequence $\left\{\sqrt{\frac{1}{2}}, \sqrt{\frac{16}{15}}, 1,1, \ldots\right\}$. Evidently, it is not a contraction. (This is the same as saying that the function $K^{\ddagger}(z, w)=8+8 z \bar{w}-z^{2} \bar{w}^{2}$ is not positive definite.) The operator $W$ is similar to the forward shift $S$. Since the class $B_{1}(\mathbb{D})$ is invariant under similarity and $S \in B_{1}(\mathbb{D})$, it follows that $W$ is in it as well. However,

$$
-\frac{\partial^{2}}{\partial w \partial \bar{w}} \log K^{\ddagger}(w, w)=-\frac{8\left(8-4|w|^{2}-|w|^{4}\right)}{\left(8+8|w|^{2}-|w|^{4}\right)^{2}}, w \in \mathbb{D},
$$

is negative for $|w|<1$. Hence we have shown that $\mathcal{K}_{M^{*}}(w)=-\frac{\partial^{2}}{\partial w \partial \bar{w}} \log K(w, w) \leq \mathcal{K}_{S^{*}}(w)$, $w \in \mathbb{D}$, although $M^{*}$ is not a contraction.

This is not an isolated example, it is easy to modify this example to produce a family of examples parameterized by a real parameter.

In the following section, we discuss the case of a commuting tuple $\boldsymbol{T}=\left(T_{1}, \ldots, T_{m}\right)$ of operators in $B_{1}(\Omega), \Omega \subseteq \mathbb{C}^{m}, m \geq 1$. Even in this case, as before, it is possible to associate a holomorphic Hermitian bundle $E_{\boldsymbol{T}}$ to the operator tuple $\boldsymbol{T}$ such that the equivalence class of the commuting $m$-tuple $\boldsymbol{T}$ determines the equivalence class of the bundle $E_{\boldsymbol{T}}$ and conversely. We show that the 
co-efficient matrix $\mathrm{K}_{T}(w)$ of the curvature $(1,1)$ form $\mathcal{K}_{\boldsymbol{T}}$ of the holomorphic Hermitian vector bundle $E_{\boldsymbol{T}}$ is negative definite for each $w \in \Omega$. The negativity of the curvature provides an alternative proof of the curvature inequality given in [7].

In the third section, we show that the curvature $\mathrm{K}_{T}$ is negative definite, that is, $\left(\left(\mathbf{K}_{T}\left(w_{i}, w_{j}\right)\right)\right)$ is negative-definite for all finite subsets $\left\{w_{1}, \ldots, w_{n}\right\}$ of $\Omega$ if we impose the additional condition of "infinite divisibility" on the reproducing kernel $K$. The infinite divisibility of the kernel $K$ requires $K^{t}$ to be positive-definite for all $t>0$.

In the final section, we give several applications of the positive definiteness of the curvature function to contractivity of operators in the Cowen-Douglas class.

\section{Negativity of the CuRvature in General}

Let $\Omega$ be a bounded domain in $\mathbb{C}^{m}$. Let $\boldsymbol{T}=\left(T_{1}, \ldots, T_{m}\right)$ be a $m$-tuple of commuting operators on a separable complex Hilbert space $\mathcal{H}$. For $x \in \mathcal{H}$, let $D_{T}: \mathcal{H} \rightarrow \mathcal{H} \oplus \ldots \oplus \mathcal{H}$ be the operator defined by $D_{\boldsymbol{T}}(x)=\left(T_{1} x, \ldots, T_{m} x\right)$. For $w=\left(w_{1}, \ldots, w_{m}\right) \in \Omega$, let $\boldsymbol{T}-w$ denote the operator tuple $\left(T_{1}-w_{1}, \ldots, T_{m}-w_{m}\right)$. The joint kernel of $\boldsymbol{T}-w$ is $\cap_{j=1}^{m} \operatorname{ker}\left(T_{j}-w_{j}\right)$, which is also the kernel of the operator $D_{\boldsymbol{T}-w}$. Following [5], we say that the commuting tuple $\boldsymbol{T}$ belongs to the Cowen-Douglas class $B_{n}(\Omega)$ if $\operatorname{ran} D_{\boldsymbol{T}-w}$ is closed, dim ker $D_{\boldsymbol{T}-w}=n$ for all $w$ in $\Omega$, and the span of $\left\{\operatorname{ker} D_{T-w}: w \in \Omega\right\}$ is dense in $\mathcal{H}$. The class of the corresponding holomorphic Hermitian vector bundle

$$
E_{\boldsymbol{T}}=\left\{(w, x) \in \Omega \times \mathcal{H}: x \in \operatorname{ker} D_{\boldsymbol{T}-w}\right\}
$$

determines the class of the operator tuple $\boldsymbol{T}$. As before, if $n=1$, then the curvature of $E_{\boldsymbol{T}}$ (cf. [4, 5]) determines the unitary equivalence class of $\boldsymbol{T}$. If $\gamma$ is a non-zero holomorphic section of the holomorphic Hermitian line bundle $E_{\boldsymbol{T}}$ defined on some open subset $\Omega_{0} \subseteq \Omega$, then the curvature of the line bundle $E_{\boldsymbol{T}}$ is the $(1,1)$ form

$$
\mathcal{K}_{\boldsymbol{T}}(w)=-\sum_{i, j=1}^{m} \frac{\partial^{2} \log \|\gamma(w)\|^{2}}{\partial w_{i} \partial \bar{w}_{j}} d w_{i} \wedge d \bar{w}_{j}, w \in \Omega_{0},
$$

defined on $\Omega_{0}$. Let

$$
\mathbf{K}_{\boldsymbol{T}}(w)=\left(\left(-\frac{\partial^{2} \log \|\gamma(w)\|^{2}}{\partial w_{i} \partial \bar{w}_{j}}\right)\right)_{i, j=1}^{m}, w \in \Omega_{0},
$$

denotes the curvature matrix. In general, for a holomorphic Hermitian vector bundle, there are two well-known notions of positivity due to Nakano and Griffiths (cf. [6, page - 338]). These two notions coincide in the case of a line bundle, and one talks of positive line bundle in an unambiguous manner. The following Proposition shows that the line bundle corresponding to a commuting tuple of operators in $B_{1}(\Omega)$ is negative.

Proposition 2.1. For an operator $\boldsymbol{T}$ in $\mathrm{B}_{1}\left(\Omega^{*}\right)$, the matrix $\mathrm{K}_{\boldsymbol{T}}(w)$ is negative definite for each $w \in \Omega^{*}$.

First Proof. Fix $w_{0} \in \Omega$. As before (cf. [5]), it follows that $\boldsymbol{T}$ can be realized as $\boldsymbol{M}^{*}=$ $\left(M_{1}^{*}, \ldots, M_{m}^{*}\right)$ where $M_{i}$ is the multiplication operator by the co-ordinate function $z_{i}$ on the Hilbert space $\mathcal{H}_{K}$ of holomorphic functions on $\Omega_{0} \subseteq \Omega, w_{0} \in \Omega_{0}$, possessing a reproducing kernel $K$ with $K(w, w) \neq 0$ for $w \in \Omega_{0}$. The function

$$
K_{0}(z, w)=K\left(w_{0}, w_{0}\right)^{\frac{1}{2}} \varphi(z)^{-1} K(z, w) \overline{\varphi(w)^{-1}} K\left(w_{0}, w_{0}\right)^{\frac{1}{2}}
$$

is defined on some open neighborhood $U \times U$ of $\left(w_{0}, w_{0}\right)$, where $U$ is the open set on which $K\left(z, w_{0}\right)$ is non-zero and $\varphi(z)=K\left(z, w_{0}\right)$ is holomorphic on $U$. The kernel $K_{0}$ is said to be normalized at $w_{0}([5])$. The operator of multiplication by the holomorphic function $\varphi^{-1}$ then defines a unitary operator from the Hilbert space $\mathcal{H}_{K}$ determined by the kernel function $K$ to 
the Hilbert space $\mathcal{H}_{K_{0}}$ determined by the normalized kernel function $K_{0}$. This unitary operator intertwines the two multiplication operators on $\mathcal{H}_{K}$ and $\mathcal{H}_{K_{0}}$ respectively. Thus $\mathcal{K}_{M^{*}}\left(w_{0}\right)$ is equal to the curvature $\mathcal{K}_{\boldsymbol{M}^{(0)}}{ }^{*}\left(w_{0}\right)$ [5, Lemma 3.9], where $\boldsymbol{M}^{(0)}$ is the $m$-tuple of multiplication operator by the co-ordinate function $z_{i}$ on the Hilbert space $\mathcal{H}_{K_{0}}$. Let

$$
K_{0}(z, w)=\sum_{I, J} a_{I J}\left(z-w_{0}\right)^{I}\left(\bar{w}-\bar{w}_{0}\right)^{J}, z, w \in U, I, J \in \mathbb{Z}_{+}^{m},
$$

be the power series expansion of $K_{0}$ around the point $\left(w_{0}, w_{0}\right)$. Since $K_{0}\left(z, w_{0}\right)=1$, we have that $a_{00}=1$ and $a_{I 0}=0$ for all $I$ with $|I|>0$. Similarly, $K_{0}\left(w_{0}, z\right)=\overline{K_{0}\left(z, w_{0}\right)}$ shows that $a_{0 J}=0$ for all $J$ with $|J|>0$. Also note that if

$$
K_{0}(z, w)^{-1}=\sum_{I, J} b_{I J}\left(z-w_{0}\right)^{I}\left(\bar{w}-\bar{w}_{0}\right)^{J}, z, w \in U, I, J \in \mathbb{Z}_{+}^{m},
$$

then $b_{00}=1$ and $b_{I 0}=0=b_{0 J}$ for all $I, J$ with $|I|,|J|>0$. Since $\gamma(w)=K_{0}(\cdot, \bar{w}), w \in U^{*}:=$ $\{\bar{z}: z \in U\}$ is a section of the holomorphic Hermitian line bundle $E_{\widetilde{\boldsymbol{M}}^{*}}$ over $U^{*}$, we have

$$
\begin{aligned}
& \left.\frac{\partial^{2} \log \|\gamma(w)\|^{2}}{\partial w_{i} \partial \bar{w}_{j}}\right|_{w=w_{0}} \\
= & \left.\frac{\partial}{\partial \bar{w}_{j}}\left(K_{0}(\bar{w}, \bar{w})^{-1} \frac{\partial}{\partial w_{i}} K_{0}(\bar{w}, \bar{w})\right)\right|_{w=w_{0}} \\
= & \left.\frac{\partial}{\partial \bar{w}_{j}}\left\{\left(1+\sum_{|I|,|J| \geq 1} b_{I J}\left(\bar{w}-\bar{w}_{0}\right)^{I}\left(w-w_{0}\right)^{J}\right)\left(\sum_{|I| \geq 1,|J| \geq 0} a_{I J+\varepsilon_{i}}\left(J_{i}+1\right)\left(\bar{w}-\bar{w}_{0}\right)^{I}\left(w-w_{0}\right)^{J}\right)\right\}\right|_{w=w_{0}} \\
= & a_{\varepsilon_{j} \varepsilon_{i}}
\end{aligned}
$$

where $\varepsilon_{i}$ is the standard unit vector in $\mathbb{C}^{m}$ with 1 at the $i$-th co-ordinate and 0 elsewhere. On the other hand, we have

$$
a_{\varepsilon_{j} \varepsilon_{i}}=\left.\frac{\partial^{2} K_{0}(\bar{w}, \bar{w})}{\partial w_{i} \partial \bar{w}_{j}}\right|_{w=w_{0}}=\left.\left\langle\frac{\partial}{\partial w_{i}} K_{0}(\cdot, \bar{w}), \frac{\partial}{\partial w_{j}} K_{0}(\cdot, \bar{w})\right\rangle\right|_{w=w_{0}} .
$$

Thus for any complex constants $\alpha_{1}, \ldots, \alpha_{m}$,

$$
-\left.\sum_{i, j=1}^{m} \alpha_{i} \bar{\alpha}_{j} \frac{\partial^{2} \log \|\gamma(w)\|^{2}}{\partial w_{i} \partial \bar{w}_{j}}\right|_{w=w_{0}}=-\left.\left\|\sum_{i=1}^{m} \alpha_{i} \frac{\partial}{\partial w_{i}} K_{0}(\cdot, \bar{w})\right\|^{2}\right|_{w=w_{0}} \leq 0 .
$$

This completes the proof.

Second Proof. We show that $-\mathrm{K}_{T}(w)$ is the Gramian of a set of $n$ vectors which is explicitly exhibited below. These vectors are

$$
e_{i}(w)=K_{w} \otimes \frac{\partial}{\partial \bar{w}_{i}} K_{w}-\frac{\partial}{\partial \bar{w}_{i}} K_{w} \otimes K_{w}, 1 \leq i \leq n,
$$

in $\mathcal{H}_{K} \otimes \mathcal{H}_{K}$. Then

$$
\begin{aligned}
\left\langle e_{i}(w), e_{j}(w)\right\rangle & =\left\langle K_{w} \otimes \frac{\partial}{\partial \bar{w}_{i}} K_{w}-\frac{\partial}{\partial \bar{w}_{i}} K_{w} \otimes K_{w}, K_{w} \otimes \frac{\partial}{\partial \bar{w}_{j}} K_{w}-\frac{\partial}{\partial \bar{w}_{j}} K_{w} \otimes K_{w}\right\rangle \\
& =2\left(K(w, w) \frac{\partial^{2} K(w, w)}{\partial w_{i} \partial \bar{w}_{j}}-\frac{\partial}{\partial w_{i}} K(w, w) \frac{\partial}{\partial \bar{w}_{j}} K(w, w)\right) .
\end{aligned}
$$

Thus

$$
\begin{aligned}
\left.\frac{\partial^{2} \log \|\gamma(w)\|^{2}}{\partial w_{i} \partial \bar{w}_{j}}\right|_{w=w_{0}} & =\left.\frac{K(w, w) \frac{\partial^{2} K(w, w)}{\partial w_{i} \partial \bar{w}_{j}}-\frac{\partial}{\partial w_{i}} K(w, w) \frac{\partial}{\partial \bar{w}_{j}} K(w, w)}{K(w, w)^{2}}\right|_{w=w_{0}} \\
& =\frac{\left\langle e_{i}\left(w_{0}\right), e_{j}\left(w_{0}\right)\right\rangle}{2 K\left(w_{0}, w_{0}\right)^{2}}
\end{aligned}
$$


This completes the proof.

A commuting tuple of operators $\boldsymbol{T}=\left(T_{1}, \ldots, T_{m}\right)$ is said to be a row contraction if $\sum_{i=1}^{m} T_{i} T_{i}^{*} \leq$ I. The following characterization of row contractions is well known (cf. [7, Corollary 2]).

Lemma 2.2. Let $\mathbb{B}^{m}$ be the unit ball in $\mathbb{C}^{m}$ and $\boldsymbol{M}=\left(M_{1}, \ldots, M_{m}\right)$ be $m$-tuples of multiplication operator on reproducing kernel Hilbert space with reproducing kernel $K$. Then $\boldsymbol{M}$ is a row contraction if and only if $(1-\langle z, w\rangle) K(z, w)$ is positive definite.

Let $\boldsymbol{R}_{m}^{*}$ be the adjoint of the joint weighted shift operator on the Drury-Arveson space $H_{m}^{2}$. This is the commuting tuple $\left(M_{1}^{*}, \ldots, M_{m}^{*}\right)$ on $H_{m}^{2}$ which is determined by the reproducing kernel $\frac{1}{1-\langle z, w\rangle}, z=\left(z_{1}, \ldots, z_{m}\right), w=\left(w_{1}, \ldots, w_{m}\right) \in \mathbb{B}^{m}$. As in Remark 1.4, using Proposition 2.1] and Lemma 2.2, we obtain a version of curvature inequality for the multi-variate case. It appeared earlier in [7] with a different proof.

Corollary 2.3. If $\boldsymbol{T}=\left(T_{1}, \ldots, T_{m}\right)$ is a row contraction in $B_{1}\left(\mathbb{B}^{m}\right)$, then $\mathrm{K}_{\boldsymbol{R}_{m}^{*}}(w)-\mathrm{K}_{\boldsymbol{T}}(w)$ is positive for each $w$ in the unit ball $\mathbb{B}^{m}$.

\section{INFINITE DIVISIBILITY AND CURVATURE INEQUALITY}

Starting with a positive definite kernel $K$ on a bounded domain $\Omega$ in $\mathbb{C}^{m}$, it is possible to construct several new positive definite kernel functions. For instance, if $K$ is positive definite then the kernel $K^{n}, n \in \mathbb{N}$, is also positive definite. Indeed, a positive definite kernel $K$ is said to be infinitely divisible if for all $t>0$, the kernel $K^{t}$ is also positive definite. While the Bergman kernel for the Euclidean ball is easily seen to be infinitely divisible, it is not infinitely divisible for the unit ball (with respect to the operator norm) of the $n \times n$ matrices. We give the details for $n=2$ in the final Section of this note. The following Lemma shows that if $K$ is positive definite then the matrix valued kernel $\left(\left(\frac{\partial^{2}}{\partial z_{i} \partial \bar{w}_{j}} K(z, w)\right)\right)_{i, j=1}^{m}$ is positive definite as well.

Lemma 3.1. For any bounded domain $\Omega$ in $\mathbb{C}^{m}$, if $K$ defines a positive definite kernel on $\Omega$, then $-\mathrm{K}(z, w)=\left(\left(\frac{\partial^{2}}{\partial z_{i} \partial \bar{w}_{j}} K(z, w)\right)\right)_{i, j=1}^{m}$ is a positive definite kernel on $\Omega$.

Proof. Let $\xi_{i}=\left(\xi_{i}(1), \ldots, \xi_{i}(m)\right), 1 \leq i \leq m$, be vectors in $\mathbb{C}^{m}$ and $u_{1}, \ldots, u_{n}$ be an arbitrary set of $n$ points in $\Omega$. Since $\bar{\partial}_{i} K_{w}$ belongs to $\mathcal{H}_{K}$, as shown in [5], it follows that

$$
\begin{aligned}
\sum_{i, j}^{n}\left\langle-\mathrm{K}\left(u_{i}, u_{j}\right) \xi_{j}, \xi_{i}\right\rangle_{\mathbb{C}^{m}} & =\sum_{i, j}^{n} \sum_{k, l}^{m}\left(\frac{\partial^{2}}{\partial w_{k} \partial \bar{w}_{l}} K\right)\left(u_{i}, u_{j}\right) \xi_{j}(l) \overline{\xi_{i}(k)} \\
& =\sum_{i, j}^{n} \sum_{k, l}^{m}\left\langle\frac{\partial}{\partial \bar{w}_{l}} K_{u_{j}}, \frac{\partial}{\partial \bar{w}_{k}} K_{u_{i}}\right\rangle_{\mathcal{H}_{K}} \xi_{j}(l) \overline{\xi_{i}(k)} \\
& =\left\|\sum_{i}^{n} \sum_{k}^{m} \xi_{i}(k) \frac{\partial}{\partial \bar{w}_{k}} K_{u_{i}}\right\|_{\mathcal{H}_{K}}^{2} \\
& \geq 0
\end{aligned}
$$

This completes the proof.

Remark 3.2. Even in the case of one variable, the proof of the Lemma given is interesting. In fact, this motivates the proof of the main theorem (Theorem 3.6 ) in one direction. In particular, it says that if $K$ is a positive definite kernel on a bounded domain $\Omega \subset \mathbb{C}$, then $\left(\frac{\partial^{2}}{\partial z \partial \bar{w}} K\right)(z, w)$ is also a positive definite kernel on $\Omega$. Without loss of generality, assume that 0 is in $\Omega$ and let $K(z, w)=\sum_{m, n}^{\infty} a_{m n} z^{m} \bar{w}^{n}$ be the power series expansion of $K$ around 0 . It is shown in [5, 
Lemma 4.1 and 4.3] that the positivity of the kernel $K$ is equivalent to the positivity of the matrix of Taylor co-efficients of $K$ at 0 , namely,

$$
H_{n}(0 ; K):=\left(\begin{array}{ccccc}
a_{00} & a_{01} & a_{02} & \cdots & a_{0 n} \\
a_{10} & a_{11} & a_{12} & \cdots & a_{1 n} \\
\vdots & \vdots & \vdots & \ddots & \vdots \\
a_{n 0} & a_{n 1} & a_{n 2} & \cdots & a_{n n}
\end{array}\right)
$$

for each $n \in \mathbb{Z}_{+}$. The function $\frac{\partial^{2}}{\partial z \partial \bar{w}} K(z, w)$ admits the expansion

$$
\sum_{m, n=0}^{\infty}(m+1)(n+1) a_{(m+1)(n+1)} z^{m} \bar{w}^{n}
$$

Therefore, for $n \in \mathbb{N}$,

$$
H_{n-1}\left(0 ; \frac{\partial^{2}}{\partial z \partial \bar{w}} K\right)=\left(\begin{array}{cccc}
a_{11} & 2 a_{12} & \cdots & n a_{1 n} \\
2 a_{21} & 4 a_{22} & \cdots & 2 n a_{2 n} \\
\vdots & \vdots & \ddots & \vdots \\
n a_{n 1} & 2 n a_{n 2} & \cdots & n^{2} a_{n n}
\end{array}\right) .
$$

Clearly, for $n \in \mathbb{N}$, we have

$$
\left(\begin{array}{cc}
0_{1 \times 1} & 0_{1 \times n} \\
0_{n \times 1} & H_{n-1}\left(0 ; \frac{\partial^{2}}{\partial z \partial \bar{w}} K\right)
\end{array}\right)=D\left(H_{n}(0 ; K)\right) D
$$

where $D: \mathbb{C}^{n+1} \rightarrow \mathbb{C}^{n+1}$ is the linear map which is diagonal and is determined by the sequence $\{0,1, \ldots, k, \ldots, n\}$. It therefore follows that $H_{n}\left(0 ; \frac{\partial^{2}}{\partial z \partial \bar{w}} K\right)$ is positive definite for all $n \in \mathbb{Z}_{+}$. Consequently, $\frac{\partial^{2}}{\partial z \partial \bar{w}} K$ is a positive definite kernel.

The following Lemma encodes a way to extract scalar valued positive definite kernel from the matrix valued one.

Lemma 3.3. If $K$ is a $n \times n$ matrix valued positive definite kernel on a bounded domain $\Omega \subset \mathbb{C}^{m}$, then for every $\zeta \in \mathbb{C}^{n},\langle K(z, w) \zeta, \zeta\rangle_{\mathbb{C}^{n}}$ is also a positive definite kernel on $\Omega$.

Proof. Let $K_{\zeta}(z, w)=\langle K(z, w) \zeta, \zeta\rangle_{\mathbb{C}^{n}}$. Let $u_{1}, \ldots, u_{l}$ be $l$ points in $\Omega$ and $\alpha_{i}, 1 \leq i \leq l$, be scalars in $\mathbb{C}$. From [5], it follows that

$$
\begin{aligned}
\sum_{i, j}^{l} \alpha_{i} K_{\zeta}\left(u_{i}, u_{j}\right) \bar{\alpha}_{j} & =\sum_{i, j}^{l} \alpha_{i} \bar{\alpha}_{j}\left\langle K\left(\cdot, u_{j}\right) \zeta, K\left(\cdot, u_{i}\right) \zeta\right\rangle_{\mathcal{H}_{K}} \\
& =\left\|\sum_{j}^{l} \bar{\alpha}_{j} K\left(\cdot, u_{j}\right) \zeta\right\|_{\mathcal{H}_{K}}^{2} \\
& \geq 0
\end{aligned}
$$

This completes the proof.

Definition 3.4. Let $G$ be a real analytic function of $w, \bar{w}$ for $w$ in some open connected subset $\Omega$ of $\mathbb{C}^{n}$. Polarizing $G$, we obtain a (unique) new function $\widetilde{G}$ defined on $\Omega \times \Omega$ which is holomorphic in the first variable and anti-holomorphic in the second and restricts to $G$ on the diagonal set $\{(w, w): w \in \Omega\}$, that is, $\tilde{G}(w, w)=G(w, w), w \in \Omega$. If the function $\tilde{G}$ is also positive definite, that is, the $n \times n$ matrix $\left(\left(\widetilde{G}\left(w_{i}, \bar{w}_{j}\right)\right)\right)$ is positive definite for all finite subsets $\left\{w_{1}, \ldots, w_{n}\right\}$ of $\Omega$, then we say that $G$ is a positive definite function on $\Omega$. 
The curvature $\mathcal{K}$ of a line bundle is a real analytic function. We have shown that $-\mathrm{K}(w)$, $w \in \Omega \subset \mathbb{C}^{m}$, is positive definite. However, the following example shows that $-\mathrm{K}(w)$ need not be a positive definite function, that is, $-\widetilde{\mathrm{K}}(w)$ need not be positive definite! We adopt the convention that the positive definiteness of the real analytic function $-\mathrm{K}(w)$ is the same as the positive definiteness of the Hermitian function $-\widetilde{\mathrm{K}}(w)$.

Example 3.5. Let $K(z, w)=1+\sum_{i=1}^{\infty} a_{i} z^{i} \bar{w}^{i}$ be a positive definite kernel on the unit disc $\mathbb{D}$. The kernel $K$ then admits a power series expansion some small neighborhood of 0 . Consequently, we have

$$
\begin{aligned}
\log K(z, w) & =\log \left(1+\sum_{i=1}^{\infty} a_{i} z^{i} \bar{w}^{i}\right) \\
& =\sum_{i=1}^{\infty} a_{i} z^{i} \bar{w}^{i}-\frac{\left(\sum_{i=1}^{\infty} a_{i} z^{i} \bar{w}^{i}\right)^{2}}{2}+\frac{\left(\sum_{i=1}^{\infty} a_{i} z^{i} \bar{w}^{i}\right)^{3}}{3}-\ldots \\
& =a_{1} z \bar{w}+\left(a_{2}-\frac{a_{1}^{2}}{2}\right) z^{2} \bar{w}^{2}+\left(a_{3}-a_{1} a_{2}+\frac{a_{1}^{3}}{3}\right) z^{3} \bar{w}^{3}+\ldots
\end{aligned}
$$

It follows that

$$
\left(\frac{\partial^{2}}{\partial z \partial \bar{w}} \log K\right)(z, w)=a_{1}+4\left(a_{2}-\frac{a_{1}^{2}}{2}\right) z \bar{w}+9\left(a_{3}-a_{1} a_{2}+\frac{a_{1}^{3}}{3}\right) z^{2} \bar{w}^{2}+\ldots
$$

Thus if we choose $0<a_{i}, i \in \mathbb{N}$, such that $a_{2}<\frac{a_{1}^{2}}{2}$, then from [5, Lemma 4.1 and 4.3], it follows that $\frac{\partial^{2}}{\partial z \partial \bar{w}} \log K$ is not positive definite.

However we note, for instance, that if $K$ is the function $1+z \bar{w}+\frac{1}{4} z^{2} \bar{w}^{2}+\sum_{i=3}^{\infty} z^{i} \bar{w}^{i}$, then

$$
K^{t}(z, w)=1+t z \bar{w}+\frac{t(2 t-1)}{4} z^{2} \bar{w}^{2}+\cdots
$$

is not positive definite for $t<\frac{1}{2}$.

It is therefore natural to ask if assuming that $K$ is infinitely divisible is both necessary and sufficient for positive definiteness of the curvature function $-K$. The following Theorem provides an affirmative answer.

Theorem 3.6. Let $\Omega$ be a domain in $\mathbb{C}^{m}$ and $K$ be a positive real analytic function on $\Omega \times$ $\Omega$. If $K$ is infinitely divisible then there exist a domain $\Omega_{0} \subseteq \Omega$ such that the curvature matrix $\left(\left(\frac{\partial^{2}}{\partial w_{i} \partial \bar{w}_{j}} \log K\right)\right)_{i, j=1}^{m}$ is positive definite function on $\Omega_{0}$. Conversely, if the function $\left(\left(\frac{\partial^{2}}{\partial w_{i} \partial \bar{w}_{j}} \log \hat{K}\right)\right)_{i, j=1}^{m}$ is a positive definite on $\Omega$, then there exist a neighborhood $\Omega_{0} \subseteq \Omega$ of $w_{0}$ and a infinitely divisible kernel $K$ on $\Omega_{0} \times \Omega_{0}$ such that $K(w, w)=\hat{K}(w, w)$, for all $w \in \Omega_{0}$.

Proof. For each $t>0$, assume that $K^{t}$ is positive definite on $\Omega$. This is the same as the positive definiteness of $\exp t \log K, t>0$. Clearly $t^{-1}(\exp t \log K-1)$ is conditionally positive definite (An Hermitian kernel $L$ is said to be conditionally positive definite if for every $n \in \mathbb{N}$ and for every choice $n$ points $w_{1}, \ldots, w_{n}$ and complex scalars $\alpha_{1}, \ldots, \alpha_{n}$ with $\sum_{i=1}^{n} \alpha_{i}=0$, the inequality $\sum_{i, j=1}^{n} \alpha_{i} \bar{\alpha}_{j} L\left(w_{i}, w_{j}\right) \geq 0$ holds). By letting $t$ tend to 0 , it follows that $\log K$ is conditionally positive definite. Hence at an arbitrary point in $\Omega$, in particular at $w_{0}$, the kernel

$$
L_{w_{0}}(z, w)=\log K(z, w)-\log K\left(z, w_{0}\right)-\log K\left(w_{0}, w\right)+\log K\left(w_{0}, w_{0}\right)
$$

is positive definite. This is essentially the Lemma 1.7 in [9]. From Lemma 3.1, it follows that the matrix $\left(\left(\frac{\partial^{2}}{\partial w_{i} \partial \bar{w}_{j}} L_{w_{0}}\right)\right)$ is positive definite on $\Omega$. Note that there exist a neighborhood $\Omega_{0} \subseteq \Omega$ of $w_{0}$ such that $\log K\left(z, w_{0}\right)$ is holomorphic on $\Omega_{0}$. Hence from the equation above, the curvature matrix $\left(\left(\frac{\partial^{2}}{\partial w_{i} \partial \bar{w}_{j}} \log K\right)\right)$ is positive definite on $\Omega_{0}$. This proves the Theorem in the forward direction. 
For the other direction, without loss of generality, assume that $w_{0}=0$. Let $\mathrm{K}(z, w)$ be the function obtained by polarizing the real analytic $m \times m$ matrix valued function

$$
\left(\left(\frac{\partial^{2}}{\partial w_{i} \partial \bar{w}_{j}} \log \hat{K}(w, w)\right)\right)_{i, j=1}^{m}
$$

defined on some bounded domain $\Omega$ in $\mathbb{C}^{m}$. Suppose that $\log \hat{K}$ has the power series expansion $\sum a_{I J} z^{I} \bar{w}^{J}$, where the sum is over all multi-indices $I, J$ of length $m$ and $z^{I}=z_{1}^{i_{1}} \cdots z_{m}^{i_{m}}, \bar{w}^{J}=$ $\bar{w}_{1}^{j_{1}} \cdots \bar{w}_{m}^{j_{m}}$. Then

$$
\mathrm{K}(z, w)=\sum_{I, J} a_{I J}\left(\left(A_{I J}(k, \ell) z^{I-\epsilon_{k}} \bar{w}^{J-\epsilon_{\ell}}\right)\right),
$$

where $\left.A_{I J}(k, \ell)=\left(i_{k} j_{\ell}\right)\right)_{k, \ell=1}^{m}$ and the sum is again over all multi-indices $I, J$ of size $m$. Clearly, $A_{I J}$ can be written as the product $D(I) E_{m} D(J)$, where $D(I)$ and $D(J)$ be the $m \times m$ diagonal matrices with $\left(i_{1}, \ldots, i_{m}\right)$ and $\left(j_{1}, \ldots, j_{m}\right)$ on their diagonal respectively, and $E_{m}$ is the $m \times m$ matrix all of whose entries are 1.

Let $D(z)$ be the holomorphic function on $\Omega$ taking values in the $m \times m$ diagonal matrices which has $z_{i}$ in the $(i, i)$ position for $z:=\left(z_{1}, z_{2}, \ldots, z_{m}\right) \in \Omega$. If the function $\mathrm{K}$ is assumed to be positive definite then

$$
\widetilde{\mathrm{K}}(z, w):=D(z) \mathrm{K}(z, w) D(\bar{w})=\sum_{I, J} a_{I J} D(I) E_{m} D(J) z^{I} \bar{w}^{J}
$$

is positive definite on $\Omega_{0}$.

Let $\Lambda(I)=\left\{k: 1 \leq k \leq m\right.$ and $\left.i_{k} \neq 0\right\}$. Consider the $m \times m$ matrix $E(I, J)$ defined below:

$$
E(I, J)_{i j}= \begin{cases}1 & \text { if } i \in \Lambda(I) \text { and } j \in \Lambda(J), \\ 0 & \text { otherwise. }\end{cases}
$$

Note that if $\Lambda(I)=\Lambda(J)=\{1, \ldots, m\}$, then $E(I, J)=E_{m}$. Consider the function on $\Omega_{0} \times \Omega_{0}$, defined by

$$
\widehat{\widetilde{\mathrm{K}}}(z, w)=\sum_{I, J \neq 0} a_{I J} \frac{E(I, J)}{c(I) c(J)} z^{I} \bar{w}^{J}
$$

where $c(I)$ denotes the cardinality of the set $\Lambda(I)$. We will prove that $\widehat{\widetilde{\mathrm{K}}}$ is a positive definite kernel on $\Omega_{0}$. To facilitate the proof, we need to fix some notations.

Let $\delta$ be a multi-index of size $m$. Also let $p(\delta)=\prod_{j=1}^{m}\left(\delta_{j}+1\right)$ which is the number of multiindices $I \leq \delta$, that is, $i_{l} \leq \delta_{l}, 1 \leq l \leq m$. As par the notation in [5], given a function $L$ on a domain $U \times U$ which is holomorphic in the first variable and antiholomorphic in the second, let $H_{\delta}\left(w_{0} ; L\right)$ be the $p(\delta) \times p(\delta)$ matrix whose $(I, J)$-entry is $\frac{\partial^{I} \bar{\partial}^{J} L\left(w_{0}, w_{0}\right)}{I ! J !}, 0 \leq I, J \leq \delta$. For convenience, one uses the colexicographic order to write down the matrix, that is, $I \leq_{c} J$ if and only if $\left(i_{m}<j_{m}\right)$ or $\left(i_{m}=j_{m}\right.$ and $\left.i_{m-1}<j_{m-1}\right)$ or $\ldots$ or $\left(i_{m}=j_{m}\right.$ and $\ldots i_{2}=j_{2}$ and $\left.i_{1}<j_{1}\right)$ or $I=J$.

Let $D(I)^{\sharp}$ be the diagonal matrix with the diagonal entry $D(I)_{\ell \ell}^{\sharp}$ equal to $\frac{1}{i_{\ell}}$ or 0 according as $i_{\ell}$ is non-zero or zero. Using this notation, we have

$$
D(I)^{\sharp} D(I) E_{m} D(J) D(J)^{\sharp}=E(I, J) .
$$

Let $A_{\delta}$ be the block diagonal matrix, written in the colexicographic ordering, of the form

$$
\left(A_{\delta}\right)_{I J}= \begin{cases}\frac{D(I)^{\sharp}}{c(I)} & \text { if } I=J(\neq 0) \\ 0 & \text { otherwise. }\end{cases}
$$

Therefore, in this setup, for any multi-index $\delta$, we have

$$
H_{\delta}(0 ; \widehat{\widetilde{\mathrm{K}}})=A_{\delta} H_{\delta}(0 ; \widetilde{\mathrm{K}}) A_{\delta}^{*} .
$$


Clearly $H_{\delta}(0 ; \widehat{\widehat{\mathrm{K}}})$ is positive definite since $H_{\delta}(0 ; \widetilde{\mathrm{K}})$ is so by [5, Lemma 4.1]. Thus from [5, Lemma 4.3], it follows that $\widehat{\widehat{K}}$ is a positive definite kernel.

Let $K_{0}$ be the scalar function on $\Omega_{0} \times \Omega_{0}$ defined by

$$
K_{0}(z, w):=\sum a_{I J} z^{I} \bar{w}^{J},
$$

where the sum is over all pairs $(I, J)$ excluding those of the form $(I, 0)$ and $(0, J)$. From Lemma 3.3. it follows that the function $K_{0}$ is positive definite since it is of the form $\langle\widehat{\widetilde{\mathrm{K}}}(z, w) \mathbf{1}, \mathbf{1}\rangle$, $\mathbf{1}=(1, \ldots, 1)$. It is evident that

$$
\left(\left(\left(\frac{\partial^{2}}{\partial w_{i} \partial \bar{w}_{j}} K_{0}\right)(w, w)\right)\right)=\mathrm{K}(w, w),
$$

that is,

$$
\frac{\partial^{2}}{\partial w_{i} \partial \bar{w}_{j}}\left(\log \hat{K}-K_{0}(w, w)\right)=0,1 \leq i, j \leq m, w \in \Omega_{0} .
$$

Therefore, $\log \hat{K}-K_{0}$ is a real pluriharmonic function on $\Omega_{0}$ and hence there exist a holomorphic function $\phi$ such that

$$
\log \hat{K}(w, w)-K_{0}(w, w)=(\Re \phi)(w):=\frac{\phi(w)+\overline{\phi(w)}}{2} .
$$

(Alternatively, since $\log \hat{K}$ is real analytic, it follows that

$$
\sum_{I, J} a_{I J} w^{I} \bar{w}^{J}=\sum_{I, J} \bar{a}_{I J} w^{J} \bar{w}^{I}
$$

Equating coefficients, we get $a_{I J}=\bar{a}_{J I}$ for all multi-indices $I, J$. In particular, we have $a_{I 0}=\overline{a_{0 I}}$ for all multi-indices $I$. The power series

$$
\left(a_{00} / 2\right)+\sum_{I} a_{I 0} z^{I}
$$

defines a holomorphic function $\psi$ on $\Omega_{0}$ such that $\log \hat{K}(w, w)-K_{0}(w, w)=\psi(w)+\overline{\psi(w)}$. Thus

$$
\hat{K}(w, w)=\exp \left(\frac{\phi(w)}{2}\right) \exp \left(K_{0}(w, w)\right) \exp \left(\frac{\overline{\phi(w)}}{2}\right), w \in \Omega_{0} .
$$

Let $K: \Omega_{0} \times \Omega_{0} \rightarrow \mathbb{C}$ be the function defined by the rule

$$
K(z, w)=\exp \left(\frac{\phi(z)}{2}\right) \exp \left(K_{0}(z, w)\right) \exp \left(\overline{\frac{\phi(w)}{2}}\right) .
$$

For $t>0$, we then have

$$
K^{t}(z, w)=\exp \left(t \frac{\phi(z)}{2}\right) \exp \left(t K_{0}(z, w)\right) \exp \left(t \frac{\overline{\phi(w)}}{2}\right), z, w \in \Omega_{0} .
$$

By construction $K(w, w)=\hat{K}(w, w), w \in \Omega_{0}$. Since $K_{0}$ is a positive definite kernel as shown above, it follows from [9, Lemma 1.6] that $\exp \left(t K_{0}\right)$ is a positive definite kernel for all $t>0$ and therefore $K^{t}$ is a positive definite on $\Omega_{0}$ for all $t>0$ completing the proof of the converse.

\section{Applications}

Let $\boldsymbol{M}^{*}$ be the adjoint of the commuting tuple of multiplication operators acting on the Hilbert space $\mathcal{H}_{K} \subseteq \mathcal{O}(\Omega)$. Fix a positive definite kernel $\mathfrak{K}$ on $\Omega$. Let us say that $\boldsymbol{M}$ is infinitely divisible with respect to $\mathfrak{K}$ if $\mathfrak{K}(z, w)^{-1} K(z, w)$ is infinitely divisible in some open subset $\Omega_{0}$ of $\Omega$. As an immediate application of Theorem [3.6, we obtain : 
Theorem 4.1. Assume that the adjoint $\boldsymbol{M}^{*}$ of the multiplication operator $\boldsymbol{M}$ on the reproducing kernel Hilbert space $\mathcal{H}_{K}$ belongs to $B_{1}(\Omega)$. The function $\left(\frac{\partial^{2}}{\partial w_{i} \partial \bar{w}_{j}} \log \left(\mathfrak{K}(w, w)^{-1} K(w, w)\right)\right)$ is positive definite, if and only if the multiplication operator $\boldsymbol{M}$ is infinitely divisible with respect to $\mathfrak{K}$.

If $K$ is a positive definite kernel on $\mathbb{D}$ such that $(1-z \bar{w}) K(z, w)$ is infinitely divisible then we say that $M^{*}$ on $\mathcal{H}_{K}$ is a infinitely divisible contraction.

Here is an example showing that a contraction need not be infinitely divisible. Take

$$
\begin{aligned}
K(z, w) & =(1-z \bar{w})^{-1}\left(1+z \bar{w}+\frac{1}{4} z^{2} \bar{w}^{2}+\sum_{n=3}^{\infty} z^{n} \bar{w}^{n}\right) \\
& =1+2 z \bar{w}+\sum_{n=2}^{\infty}\left(n+\frac{1}{4}\right) z^{n} \bar{w}^{n} .
\end{aligned}
$$

Clearly $K$ defines the positive definite kernel on $\mathbb{D}$. Since $(1-z \bar{w}) K(z, w)$ is also positive definite, it follows that the adjoint of multiplication operator $M^{*}$ on $\mathcal{H}_{K}$ is contractive. But

$$
((1-z \bar{w}) K(z, w))^{t}=1+t z \bar{w}+\frac{t(2 t-1)}{4} z^{2} \bar{w}^{2}+\cdots
$$

is not positive definite for $t<\frac{1}{2}$ as was pointed out earlier. Hence $M^{*}$ is not infinitely divisible contraction on $\mathcal{H}_{K}$.

The following Corollary is a characterization of infinitely divisible contractions in the CowenDouglas class $B_{1}(\mathbb{D})$ completing the study begun in 8 . Here, for two real analytic functions $G_{1}$ and $G_{2}$ on a domain $\Omega \subset \mathbb{C}^{m}, G_{1}(w) \preceq G_{2}(w), w \in \Omega$, means $G_{2}-G_{1}$ is a positive definite function on $\Omega$.

Corollary 4.2. Let $K$ be a positive definite kernel on the open unit disc $\mathbb{D}$. Assume that the adjoint $M^{*}$ of the multiplication operator $M$ on the reproducing kernel Hilbert space $\mathcal{H}_{K}$ belongs to $B_{1}(\mathbb{D})$. The function $\frac{\partial^{2}}{\partial w \partial \bar{w}} \log \left(\left(1-|w|^{2}\right) K(w, w)\right)$ is positive definite, or equivalently

$$
\mathcal{K}_{T}(w) \preceq \mathcal{K}_{S^{*}}(w), w \in \mathbb{D},
$$

if and only if the multiplication operator $M$ is an infinitely divisible contraction.

Proof. Recall Theorem [3.6, which says that the positive definiteness of

$$
\frac{\partial^{2}}{\partial w \partial \bar{w}} \log \left(\left(1-|w|^{2}\right) K(w, w)\right)
$$

is equivalent to infinite divisibility of the kernel $(1-z \bar{w}) K(z, w)$, that is, $((1-z \bar{w}) K(z, w))^{t}$ is positive definite for all $t \geq 0$.

We say that a commuting tuple of multiplication operators $\boldsymbol{M}$ is an infinitely divisible row contraction if $(1-\langle z, w\rangle) K(z, w)$ is infinitely divisible, that is, $((1-\langle z, w\rangle) K(z, w))^{t}$ is positive definite for all $t>0$.

Recall that $\boldsymbol{R}_{m}^{*}$ is the adjoint of the joint weighted shift operator on the Drury-Arveson space $H_{m}^{2}$. The following theorem is a characterization of infinitely divisible row contractions.

Corollary 4.3. Let $K$ be a positive definite kernel on the Euclidean ball $\mathbb{B}^{m}$. Assume that the adjoint $\boldsymbol{M}^{*}$ of the multiplication operator $\boldsymbol{M}$ on the reproducing kernel Hilbert space $\mathcal{H}_{K}$ belongs to $B_{1}\left(\mathbb{B}^{m}\right)$. The function $\left(\left(\frac{\partial^{2}}{\partial w_{i} \partial \bar{w}_{j}} \log ((1-\langle w, w\rangle) K(w, w))\right)\right)_{i, j=1}^{m}, w \in \mathbb{B}^{m}$, is positive definite, or equivalently

$$
\mathrm{K}_{M^{*}}(w) \preceq \mathrm{K}_{\boldsymbol{R}_{m}^{*}}(w), w \in \mathbb{B}^{m},
$$

if and only if the multiplication operator $\boldsymbol{M}$ is an infinitely divisible row contraction. 
We give one last example, namely that of the polydisc $\mathbb{D}^{m}$. In this case, we say a commuting tuple $\boldsymbol{M}$ of multiplication by the co-ordinate functions acting on the Hilbert space $\mathcal{H}_{K}$ is infinitely divisible if $(S(z, w) K(z, w))^{t}$, where $S(z, w):=\prod_{i=1}^{m}\left(1-z_{i} \bar{w}_{i}\right)^{-1}, z, w \in \mathbb{D}^{m}$, is positive definite for all $t>0$. Every commuting tuple of contractions $M^{*}$ need not be infinitely divisible. Let $\boldsymbol{S}_{m}$ be the commuting $m$ - tuple of the joint weighted shift defined on the Hardy space $H^{2}\left(\mathbb{D}^{m}\right)$.

Corollary 4.4. Let $K$ be a positive definite kernel on the polydisc $\mathbb{D}^{m}$. Assume that the adjoint $\boldsymbol{M}^{*}$ of the multiplication operator $\boldsymbol{M}$ on the reproducing kernel Hilbert space $\mathcal{H}_{K}$ belongs to $B_{1}\left(\mathbb{D}^{m}\right)$. The function $\left(\left(\frac{\partial^{2}}{\partial w_{i} \partial \bar{w}_{j}} \log (S(z, w) K(w, w))\right)\right)_{i, j=1}^{m}, w \in \mathbb{D}^{m}$, is positive definite, or equivalently

$$
\mathrm{K}_{M^{*}}(w) \preceq \mathrm{K}_{S_{m}^{*}}(w), w \in \mathbb{D}^{m},
$$

if and only if the multiplication operator $\boldsymbol{M}$ is an infinitely divisible m-tuple of contractions.

For a second application of these ideas, assume that $K$ is a positive definite kernel on $\mathbb{D}^{m}$ with the property:

$$
K_{i}(z, w)=\left(1-z_{i} \bar{w}_{i}\right)^{m} K(z, w), 1 \leq i \leq m,
$$

is infinitely divisible. Then

$$
K^{m}(z, w)=\left(\prod_{i=1}^{m}\left(1-z_{i} \bar{w}_{i}\right)\right)^{-m} \prod_{i=1}^{m} K_{i}(z, w) .
$$

It now follows that

$$
K(z, w)=S(z, w)\left(\prod_{i=1}^{m} K_{i}(z, w)\right)^{\frac{1}{m}}
$$

Let $M$ be the commuting tuple of multiplication operators on the Hilbert space $\mathcal{H}_{K}$, which is contractive since $K$ admits the Sz̈ego kernel $S$ as a factor. Clearly, the infinite divisibility of $K_{i}, 1 \leq i \leq m$, implies that $\left(\prod_{i=1}^{m} K_{i}(z, w)\right)^{\frac{1}{m}}$ is positive definite. As pointed out in [7], in consequence, for any polynomial $p$ in $m$ - variables,

$$
p\left(M_{1}, \ldots, M_{m}\right)=P_{\mathcal{S}} p\left(S_{m}\right)_{\mid \mathcal{S}},
$$

where $\mathcal{S}$ is the invariant subspace of functions vanishing on the diagonal of the Hilbert space $H_{m}^{2} \otimes \mathcal{H}_{K} \subseteq \mathcal{O}\left(\mathbb{D}^{m} \times \mathbb{D}^{m}\right)$ and $P_{\mathcal{S}}$ is the projection onto the subspace $\mathcal{S}$.

A basic question raised in the paper of Cowen and Douglas [3, Section 4] is the determination of non-degenerate holomorphic curves in the Grassmannian. Clearly, a necessary condition for this is the positive definiteness of the curvature function. Thus we have the following corollary to Theorem 3.6.

Corollary 4.5. Let $E$ be a holomorphic Hermitian vector bundle of rank 1 over a bounded domain $\Omega \subset \mathbb{C}^{m}$. If the curvature $\mathrm{K}$ of $E$ is negative definite, then there exists a Hilbert space $\mathcal{H}$ and a holomorphic map $\gamma: \Omega_{0} \rightarrow \mathcal{H}, \Omega_{0}$ open in $\Omega$, such that $E$ is isomorphic to $E_{\gamma}$, where $E_{\gamma}$ is the pullback, by the holomorphic map $\gamma: \Omega_{0} \rightarrow \mathcal{G r}(1, \mathcal{H})$, of the tautological bundle defined over $\mathcal{G} r(1, \mathcal{H})$. Moreover, the real analytic function $\langle\gamma(z), \gamma(w)\rangle$ defined on $\Omega_{0} \times \Omega_{0}$ is infinitely divisible.

We finish with an amusing application of the Lemma 1.7 in [9] which is a key ingredient in the proof of Theorem 3.6. Let $K$ be the function on the unit ball $\mathbb{B}_{2 \times 2}$ (with respect to the operator norm) of $2 \times 2$ matrices, given by the formula $K(Z, W):=\operatorname{det}\left(I-Z W^{*}\right)^{-1}, Z, W \in \mathbb{B}_{2 \times 2}$.

The kernel $K$ is normalized at 0 by definition. For $\delta=(1,0,0,3)$, the matrix

$$
\left(\left(\frac{\partial^{\alpha} \bar{\partial}^{\beta} \log K(0,0)}{\alpha ! \beta !}\right)\right)_{0 \leq \alpha, \beta \leq \delta}
$$


is diagonal with $\frac{\partial_{1} \partial_{4}^{3} \bar{\partial}_{1} \bar{\partial}_{4}^{3} \log K(0,0)}{3 ! 3 !}=-1<0$ (in fact for $|\delta| \leq 3$, the corresponding matrix is diagonal with non-negative entries). Here, $\delta \geq \mu$ if and only if $\delta_{i} \geq \mu_{i}$ for all $i \in\{1, \ldots, m\}$ and the matrix is written with respect to the colexicographic ordering. From [5, Lemma 4.1 and 4.3], it follows that $\log K$ is not positive definite. Hence Theorem 3.6 shows that the function $\operatorname{det}\left(I-Z W^{*}\right)^{-t}$ cannot be positive definite for all $t>0$. Of course, a lot more is known. Indeed, the set

$$
\left\{0<t: K(Z, W)^{t} \text { is positive definite }\right\}
$$

is explicitly determined in [2, Corollary 4.6].

\section{REFERENCES}

1. J. Agler and J. E. McCarthy, Pick interpolation and Hilbert function spaces, Graduate Studies in Mathematics, vol. 44, American Mathematical Society, Providence, RI, 2002. MR 1882259 (2003b:47001)

2. J. Arazy, A survey of invariant Hilbert spaces of analytic functions on bounded symmetric domains, Multivariable operator theory (Seattle, WA, 1993), Contemp. Math., vol. 185, Amer. Math. Soc., Providence, RI, 1995, pp. 7-65. MR 1332053 (96e:46034)

3. M. J. Cowen and R. G. Douglas, Complex geometry and operator theory, Acta Math. 141 (1978), no. 3-4, 187-261. MR MR501368 (80f:47012)

4. _ Operators possessing an open set of eigenvalues, Functions, series, operators, Vol. I, II (Budapest, 1980), Colloq. Math. Soc. János Bolyai, vol. 35, North-Holland, Amsterdam, 1983, pp. 323-341. MR MR751007 (85k:47033)

5. R. E. Curto and N. Salinas, Generalized Bergman kernels and the Cowen-Douglas theory, Amer. J. Math. 106 (1984), no. 2, 447-488. MR MR737780 (85e:47042)

6. J. P Demailly, Complex analytic and differential geometry, http://www-fourier.ujf-grenoble.fr/ demailly/manuscripts/agbook.pdf.

7. R. G. Douglas, G. Misra, and J. Sarkar, Contractive Hilbert modules and their dilations, to appear, Israel J. Math.

8. G. Misra, Curvature inequalities and extremal properties of bundle shifts, J. Operator Theory 11 (1984), no. 2, 305-317. MR 749164 (86h:47057)

9. K. R. Parthasarathy and K. Schmidt, Positive definite kernels, continuous tensor products, and central limit theorems of probability theory, Lecture Notes in Mathematics, Vol. 272, Springer-Verlag, Berlin, 1972. MR 0622034 (58 \#29849)

(Biswas) Department of Mathematics, Ben Gurion University of the Negev, Be'er Sheva 84105, ISRAEL

E-mail address: shibananda@gmail.com

(Keshari) Department of Mathematics, Indian Institute of Science, Bangalore 560012, India

E-mail address: kesharideepak@gmail.com

(Misra) Department of Mathematics, Indian Institute of Science, Bangalore 560012, India

E-mail address: gm@math.iisc.ernet.in 